International Journal of Engineering \& Technology, $7(2.25)(2018) 121-124$
International Journal of Engineering \& Technology
SPC
Website: www.sciencepubco.com/index.php/IJET
Research paper

\title{
Statistical analysis of optical coherent tomography image by segmentation methods using euclidean distant model
}

\author{
R. J. Hemalatha ${ }^{1}$, Mohandass $\mathbf{G}^{2}{ }^{2}$, Hari Krishnan $\mathbf{G}^{3}$ \\ ${ }^{1}$ Department of Biomedical Engineering, Vels Institute of Science,Technology and Advanced Studies Pallavaram, Chennai-600117, In- \\ dia \\ ${ }^{2}$ Bahrain Training Institute, Bahrain \\ ${ }^{3}$ Sree Vidyanikethan Engineering College, Tirupati, Andhra Pradesh, India \\ *Corresponding author E-mail: g.mohandass@gmail.com
}

\begin{abstract}
Optical Coherence Tomography (OCT) is a diagnostic measure of the images in retinal layers. The measure of retinal layers is done by image segmentation algorithm. In this work, algorithm of different threshold based segmentation techniques is computed in OCT image. Using the resultant image, comparison is done between the segmentation methods by statistical parameters such as noise factor of the signal peak, Normalized Absolute Error (NAE) and Average Difference (AD). Statistical analysis values are evaluated by multidimensional scaling (MDS) methods. By applying clustering methods in the multidimensional data, the Euclidean Distance model gives the relation distant between the algorithms. By this result, similarity or dissimilarity of segmentation methods was categorized.
\end{abstract}

Keywords: Segmentation; Multidimensional Scaling (MDS); Euclidean Distance Model; Statistical Image Analysis.

\section{Introduction}

Optical coherence tomography (OCT) is a type of imaging technique that visualise depth penetration of layers in biological systems for medical and clinicians purpose [7]. Central Serous Retinopathy (CSR) the diseases effects is as shown in figure 1 . The main aim of the image segmentation is to segment pixels into different regions like objects, parts and surfaces. The applications of the segmentation is in object recognition, estimation of boundaries within stereo systems, compression, editing or database look up. Segmentations of the gray level images provides surface information [8] \& [9].

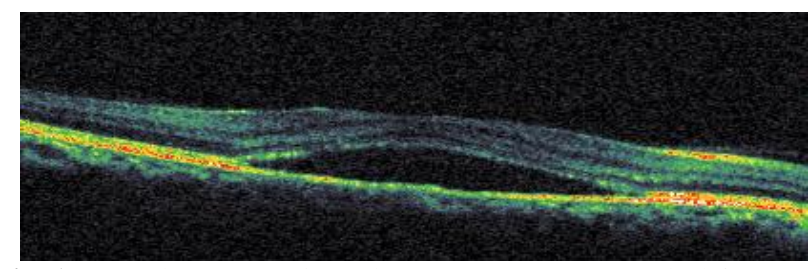

Fig. 1: Central Serous Retinopathy (CSR - OCT) - Detached from RPE Layer.

PSNR (Peak Signal to noise ratio) is a measure to determine the quality of the image before and after reconstruction. It provides the quality of a reconstructed image [10] \& [11]. Normalised absolute error techniques measure the perfect fit of image with the zero value of the original image and the computation process, segmented images. Average Difference (AD) Average Difference $(\mathrm{AD})$ is the average of difference between the reference image and original image [12] \& [13].
Multidimensional Scaling [1] is statically based on the dimensional analysis of producing a visual display of the distances between pair of methods. The quality of research, in MDS is similar to cluster analysis in identifying of similar/dissimilarity data. Euclidean Distance model give the relation distant between the algorithms and similarity or dissimilarity. In, Euclidean Distance Model, quadric lines to emphasize the clustering effect of the results such that the positive time exposure cases in the data set, The two dimensional Euclidean Distance Model for the zero time exposure/zero loss cases in the data set.

\section{Computational methods}

As discussed in the introduction, categorization on segmentation problems can be identified and analysed using the MDS data by Statistical parameters such as PSNR, NAE and AD.

\subsection{Segmentation methods by threshold techniques}

Here four categories of thresholding methods are taken according to the information they are exploiting. In this four categories, most popular and in random approach, segmentation methods are chosen are Clustering based thresholding, Entropy based thresholding, Thresholding algorithms based on attribute similarity and Local adaptive threshold method [4-6]. 


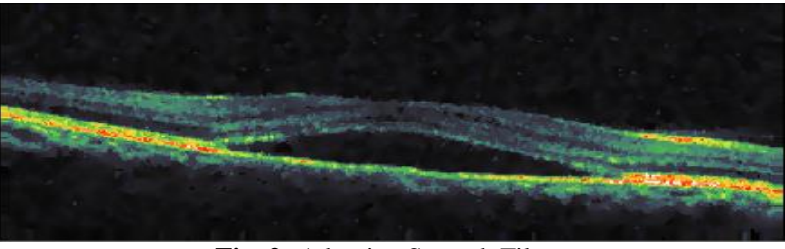

Fig. 2: Adaptive Smooth Filter.

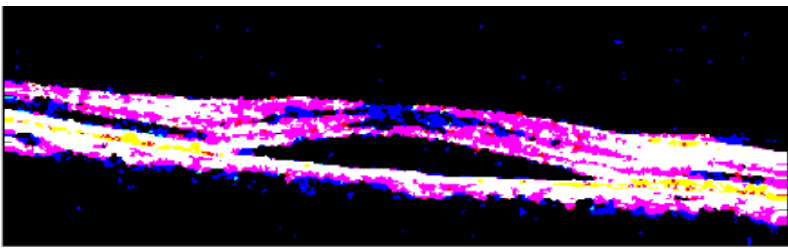

Fig. 3: Riddler Segmentation.

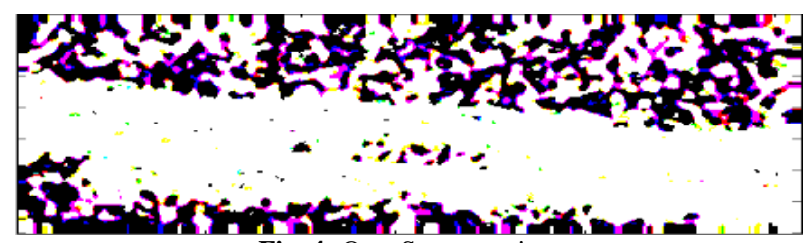

Fig. 4: Otsu Segmentation

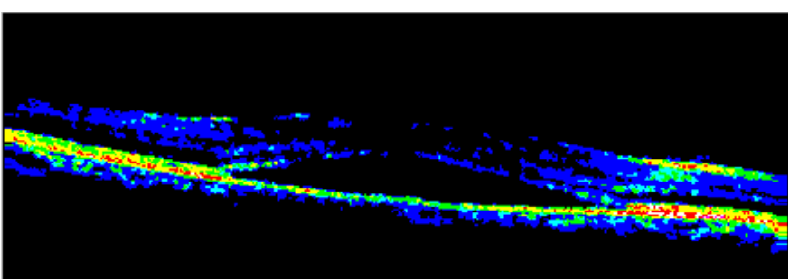

Fig. 5: Lloyd Segmentation.
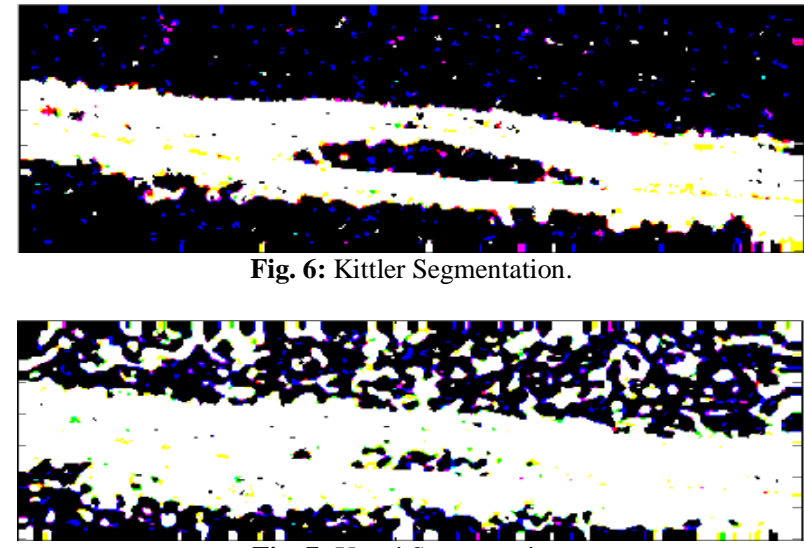

Fig. 7: Yanni Segmentation

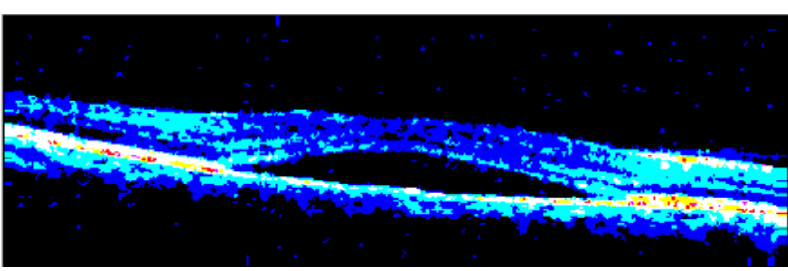

Fig. 8: Kapur Segmentation

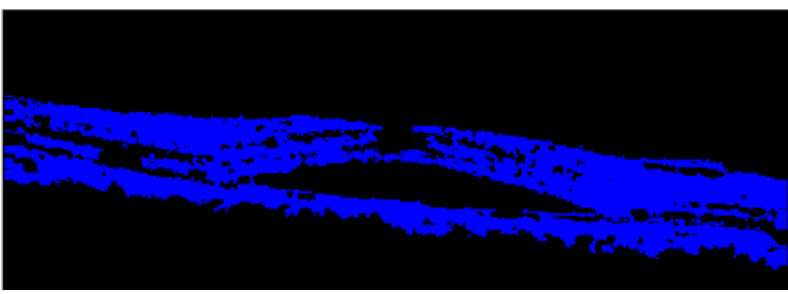

Fig. 9: Tsai Segmentation.

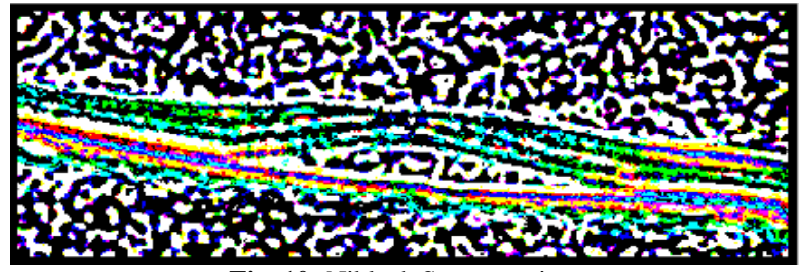

Fig. 10: Niblack Segmentation.

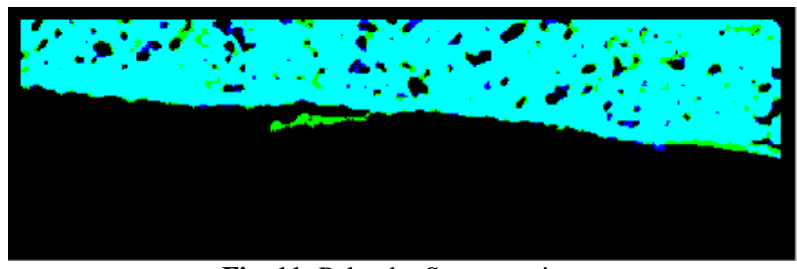

Fig. 11: Palumbo Segmentation.

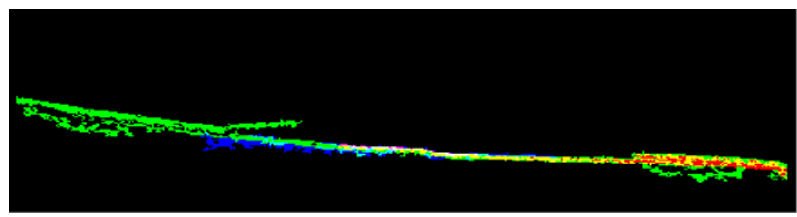

Fig. 12: Sauvola Segmentation.

\subsection{Original images and distorted images}

After applying the segmentation methods which is mentioned above in the four categories is then taken and consider as one set of data(Original set of image data) including the original image and image subjected to the computation of Adaptive smooth filter techniques. Simultaneously, same set data is taken as distorted images.Now the original set of image data is compared in segmentation methods wise with distorted image set of data.

\subsection{Multidimensional data and euclidean distant model}

Statistical Image Data analysis is done by Multidimensional scaling (MDS) to evaluate similarity or dissimilarity data which is located close together. Inter-point distances is measuring of values, variety of distance is weighted Euclidean distance model. By the OCT-CSR image, output is compared with original image and distorted images with statistical techniques. Based on the comparison, Multidimensional values are obtained for MSE, NCC and MD. Using the Pythagorean formula the distance between two points is measured and is called as Euclidean distance. The similarity and dissimilarity of any data is measured using the clustering methods.it helps in denting the distance between two instances of values. By vector coordinates, plotting is done in quadrant of axis.

\section{Result}

This relates and relevant data of redundancy represent the clustering and categorization of segmentation methods. 


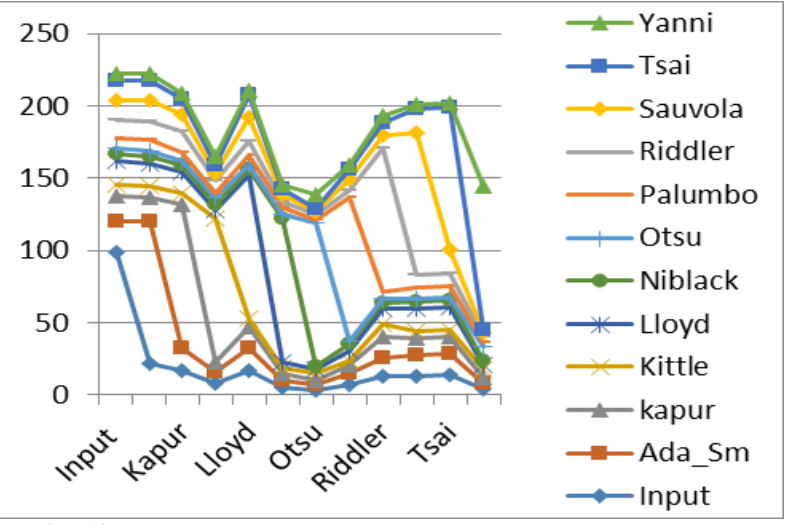

Fig. 13: Multidimensional Values of Peak Signal to Noise Ratio.

In multidimensional analysis of data by peak signal to noise ratio [Fig. 13], represent that, segmentation methods have both higher and lower range in values except the Yanni segmentation methods. In PSNR, Euclidian distant model [Fig. 14], represent that, Kittle, Yanni, Palumbo, Niblack, Otsu segmentation methods are cluster in the centre of the axis.

In multidimensional analysis of data by Normalised Absolute Error [Fig. 15], most of the segmentation methods are low range and relevant values except Yanni and Sauvola segmentation methods.

In normalised absolute error, the clustering of segmentation methods in Euclidean distant model [Fig. 16] are Kapur, Riddle, Kittle along with the input image and Adaptive smooth filter image.

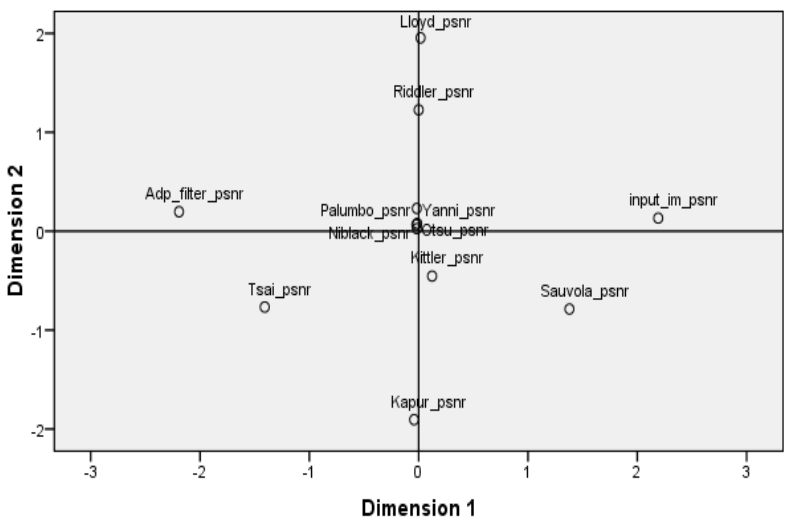

Fig. 14: Peak Signal to Noise Ratio Euclidean Distant Model.

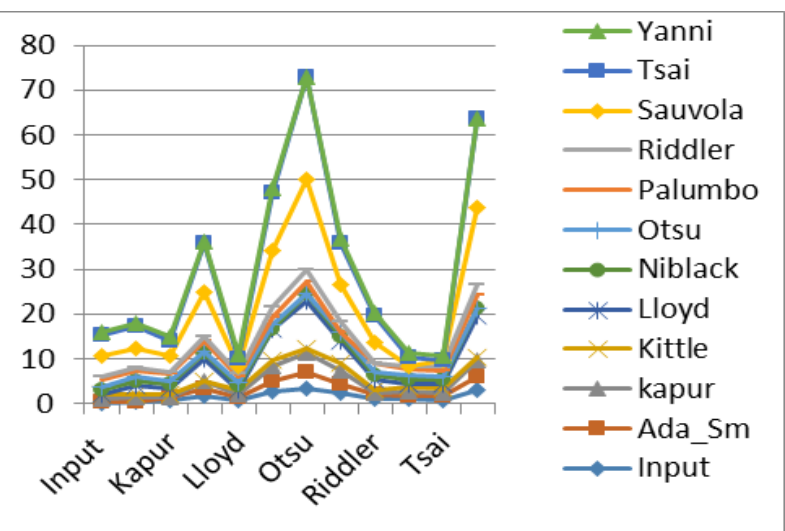

Fig. 15: Multidimensional Values of Normalized Absolute Error.

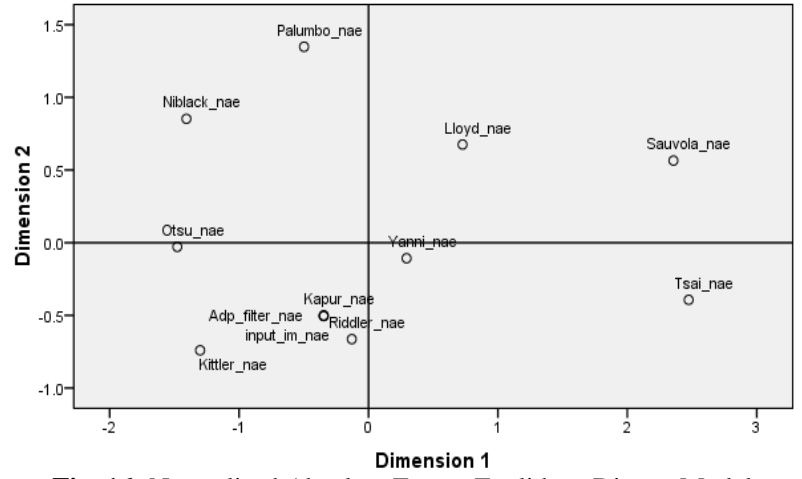

Fig. 16: Normalised Absolute Error - Euclidean Distant Model.

In multidimensional analysis of data by Average Difference [Fig. 17], the value is related and relevant with respective of the segmentation methods.

In Average difference, the clustering of segmentation methods in Euclidean distant model [Fig. 18] are related to Riddler, Lloyd, Kapur, Palumbo with the input image and Adaptive smooth filter image

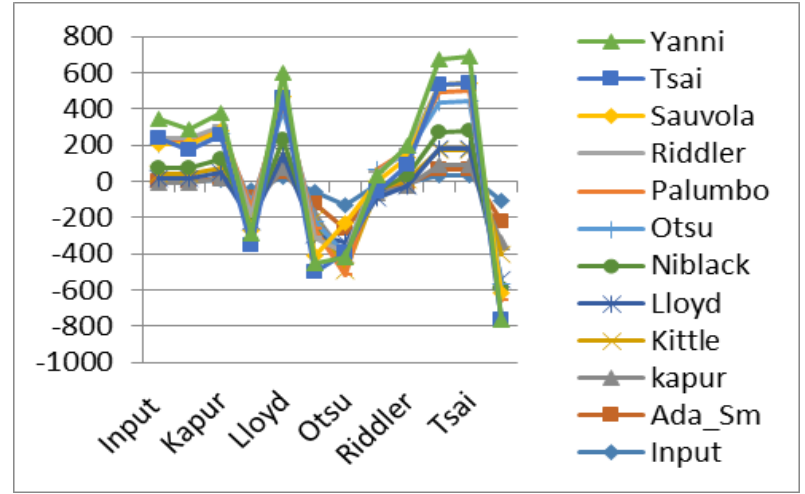

Fig. 17: Multidimensional Values of Average Difference.

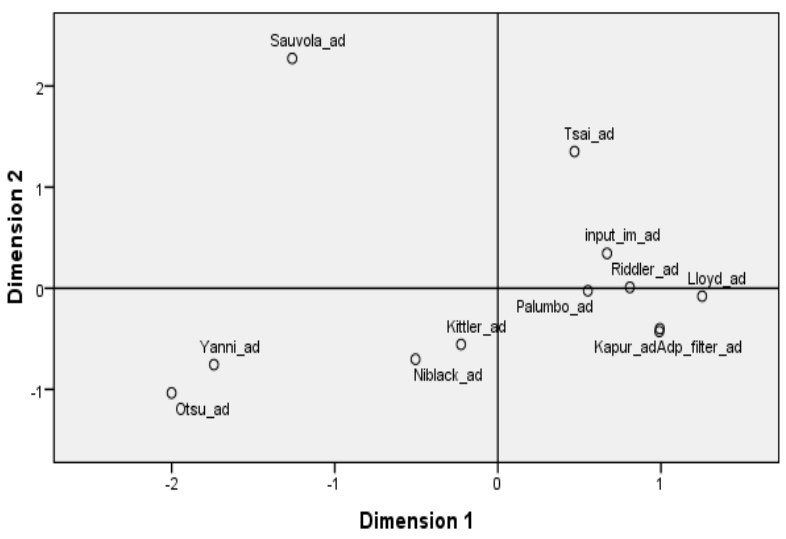

Fig. 18: Average Difference - Euclidean Distant Model.

\section{Conclusions}

In retinal layer analysis, Segmentation plays a vital role. Methods and analysis in MDS are more familiars to biological data. Here MDS improves the fitness of segmentation method between the observed Similarity and dissimilarity with the configuration of inter object distant. In Euclidean Distant Model, clustering the data is constellation by redundancy analysis. An important, in this work is to find the cluster analysis in the application to make decision regarding the numbers of cluster derived from the data. In these segmentation methods, in PSNR, NAE and AD has one cluster alone and reaming data are scattered. On analysis by the results of Centroid would be too granular, would not allow for spatial dispersion and potentially biased and sequential of analysis is done by Hierarchical methods The data of cluster segmentation 
methods are identified based on the similarly of the algorithm such that future work is progressed. This work can be extended by validation and verification using some other cluster standardization methods.

\section{Acknowledgement}

I express my gratitude for the support of OCT image from Regional Institute of Ophthalmology and Government Ophthalmic Hospital, Chennai. Dr. R Ravanan, Department of Statistics, Presidency College, Chennai for MDS models.

\section{References}

[1] J. Natalia, C. Anastasova, "A Review of Multidimensional Scaling(MDS) and its Utility in Various Psychological Domains", Tutorials in Quantitative Methods for Psychology, University of Ottawa, Vol. 5(1), p. 1-10, 2009.

[2] Sankur B., Sezginb M. Image Thresholding Techniques: a Survey over Categories. Journal of Electronic Imaging, vol. 13(1), (2004) pp. 146-165.

[3] Mehmet Sezgin, BulentSankur, "Survey over image thresholding techniques and quantitative performance evaluation", Journal of Electronic Imaging, 13(1), 146-165 Jan. 2004.

[4] T.W. Ridler, S. Calvard, Picture thresholding using an iterative selection method, IEEE Trans. System, Man and Cybernetics, SMC-8 (1978) 630-632.

[5] Otsu, N., "A Threshold Selection Method from Gray-Level Histograms," IEEE Transactions on Systems, Man, and Cybernetics, Vol. 9, No. 1, 1979, pp. 62-66.

[6] D.E. Lloyd, Automatic Target Classification Using Moment Invariant of Image Shapes, Technical Report, RAE IDN AW126, Farnborough- UK, December 1985.

[7] J. Kittler \& J. Illingworth: "Minimum Error Thresholding" Pattern Recognition, Vol 19, nr 1. 1986, pp. 41-47.

[8] M.K. Yanni, E. Horne, and A New Approach to Dynamic Thresholding, EUSIPCO-9: European Conf. on Signal Processing, Vol. 1, Edinburg, 1994, pp: 34-44.

[9] J.N. Kapur, P.K. Sahoo, A.K.C. Wong, A New Method for GrayLevel Picture Thresholding Using the Entropy of the Histogram," Graphical Models and Image Processing, 29 (1985) 273-285.

[10] W.H. Tsai, Moment-preserving thresholding: A new approach, Graphical Models and Image Processing, 19 (1985) 377-393.

[11] W. Niblack, an Introduction to Image Processing, Prentice-Hall, 1986, pp: 115-116.

[12] P.W. Palumbo, P. Swaminathan, S.N. Srihari, Document image binarization: Evaluation of algorithms, Proc. SPIE Applications of Digital Image Proc., SPIE Vol. 697, (1986), pp:278-286.

[13] J. Sauvola, M. Pietaksinen, Adaptive document image binarization, Pattern Recognition, 33 (2000) 225-236. C.K. Leung, F.K. Lam, Performance analysis of a class of iterative image thresholding algorithms, Pattern Recognition, 29(9) 1523-1530, 1996.

[14] G Mohandass, Dr. R. Anandanatarajan "Statistical Analysis of Reliability in edge detection techniques using Optical Coherent Tomography image" Indian Journal of Computer Science and Engineering (IJCSE), ISSN: 0976-5166, Vol. 4 No.2 Apr-May 2013. 